Síntese e Caracterização de um Novo Material Adsorvente Produzido a partir de Resíduos de Pneu para a Remoção de Contaminantes em Solução Aquosa

\author{
La Porta, F. A.;* Giacoppo, J. O. S.; Ramalho, T. C.
}

Rev. Virtual Quim., 2014, 6 (2), 363-373. Data de publicação na Web: 22 de dezembro de 2013

http://www.uff.br/rvq

\title{
Synthesis and Characterization of the New Adsorbent Material Produced from Waste Tires for the Removal of Contaminants in Aqueous Solution
}

\begin{abstract}
This work reports the synthesis and characterization of a resin produced from waste tire. This resin has been applied in the removal of contaminants in water. The process of obtaining the resin consists in modifying the chemical structure of the waste with the addition of sulfonic groups. For the adsorption tests were used textile dyes (cationic and anionic) and heavy metals, such as chromium, copper, iron and zinc. The findings pointed out that this resin can be a promising new material for the removal of contaminants in the aqueous medium.
\end{abstract}

Keywords: Tire; resin; adsorbent; contaminants; water.

\section{Resumo}

Este trabalho reporta a síntese e caracterização de uma resina produzida a partir de resíduos de pneu que foi aplicada na remoção de contaminantes na água. O processo de obtenção da resina consiste na modificação da estrutura química dos rejeitos com a adição de grupos sulfônicos. Para os ensaios de adsorção foram utilizados corantes têxteis (catiônico e aniônico) e metais pesados, tais como cromo, cobre, ferro e zinco. Os resultados de caracterização e adsorção da resina permitem identificar este novo material como promissor para a remoção de contaminantes no meio aquoso.

Palavras-chave: Pneu; resina; adsorvente; contaminantes; água.

\footnotetext{
* Universidade Estadual Paulista, Instituto de Química, CP 355, CEP 14801-970, Araraquara-SP, Brasil.

$M$ felipe laporta@yahoo.com.br

DOI: $\underline{10.5935 / 1984-6835.20140026}$
} 


\title{
Síntese e Caracterização de um Novo Material Adsorvente Produzido a partir de Resíduos de Pneu para a Remoção de Contaminantes em Solução Aquosa
}

\author{
Felipe A. La Porta, ${ }^{a}, *$ Juliana O. S. Giacoppo, ${ }^{b}$ Teodorico C. Ramalho ${ }^{b}$ \\ a Universidade Estadual Paulista, Instituto de Química, CP 355, CEP 14801-970, Araraquara-SP, \\ Brasil. \\ b Universidade Federal de Lavras, Departamento de Química, CP 3037, CEP 37200-000, Lavras- \\ MG, Brasil. \\ * felipe laporta@yahoo.com.br
}

Recebido em 21 de agosto de 2013. Aceito para publicação em 20 de outubro de 2013

\section{Introdução}

\section{Procedimento Experimental}

\subsection{Síntese da resina}

2.2. Caracterização do material

2.3. Teste de adsorção de corantes

2.4. Teste de adsorção de metais

\section{Resultados e Discussão}

3.1. Caracterização do material sintetizado

3.2. Capacidade de adsorção do material

\section{Conclusão}

\section{Introdução}

As borrachas ou elastômeros são materiais poliméricos que se distinguem pela capacidade de retornar rapidamente à forma e às dimensões originais. Por definição, um elastômero pode ser estirado 200 \% ou mais de seu comprimento e retornar ao tamanho original com rapidez. A borracha natural é o produto primário da coagulação do látex da seringueira e a borracha sintética é produzida a partir de derivados do petróleo. ${ }^{1} \mathrm{~A}$ partir do descobrimento do processo de vulcanização da borracha por Charles Goodyear por volta de 1836, houve uma grande revolução e avanço nos materiais a base de borracha. ${ }^{2}$ Atualmente, esse material encontra-se em nosso cotidiano sob as mais diversas formas, sendo essencial para a manufatura de inúmeros produtos.

A produção crescente de bens de consumo e artigos descartáveis feitos com os mais variados tipos de polímeros resulta no descarte de milhões de toneladas de materiais cuja degradação natural poderá 
levar vários séculos devido à sua grande estabilidade. ${ }^{3}$ Além disso, é bem conhecido que a maneira em que os resíduos estão dispostos no meio ambiente, dependendo desta forma, até substâncias reconhecidamente degradáveis, podem demorar muito se o contato com umidade e área exposta forem diminuído. Em particular, os pneus são produtos obtidos a partir do processo de vulcanização da borracha e tem como polímero fundamental o poliisopreno. ${ }^{4}$ Estima-se, por exemplo, que para a completa degradação de pneus pela natureza são necessários cerca de 600 anos. $^{5}$

Em se tratando de um tipo específico de resíduo sólido, é importante considerar que a produção mundial de pneus novos em 2005 foi de 1,32 bilhões em todo o mundo. Já o descarte de pneus usados chega a atingir, anualmente, a marca de quase 1 bilhão de unidades. ${ }^{6}$

Ao chegar ao fim de sua vida útil, os pneus podem ser descartados, reutilizados ou reciclados. Os pneus inservíveis são descartados no ambiente. Segundo a resolução CONAMA no 416, de 30 de setembro de 2009, um pneu usado que apresenta danos irreparáveis em sua estrutura, não se prestando mais à rodagem ou à reforma é denominado pneu inservível. A resolução inclui também sua destinação que deve ser feita observando a legislação vigente e normas operacionais específicas de modo a minimizar os impactos ambientais adversos, evitar danos ou riscos à saúde pública e à segurança. $^{7}$

Porém, percebe-se que o descarte final destes pneus é alvo de grande preocupação, uma vez que não havendo interesse comercial na reciclagem destes, eles passam a ser armazenados em depósitos ao ar livre, o que pode ser prejudicial ao meio ambiente e serve como recipiente para a proliferação do mosquito da dengue e outras espécies. Sua eliminação em aterros sanitários também não é aconselhável porque, dada sua forma oca, ocupa muito espaço e cria problemas de estabilidade nos aterros que não podem ser compactados convenientemente. ${ }^{8}$
Outra problemática é sua queima ao ar livre, pois como a sua composição química é complexa, essencialmente compostos de carbono, enxofre, cloro ou bromo, a combustão não controlada a baixas temperaturas leva à formação de substâncias extremamente tóxicas como, por exemplo, dioxinas. ${ }^{9}$ Além disto, a combustão deste material produz elevadíssima quantidade de matéria particulada no ar. $^{10}$

O reuso de pneus se faz através da recauchutagem, ou seja, um processo que consiste em reformar o pneu, através da substituição de sua banda de rodagem e dos seus ombros. E por último, a reciclagem é vista como um conjunto de tecnologias que visam aproveitar os resíduos gerados de alguma forma, e se possível, em escala industrial. ${ }^{6}$

Algumas das aplicações que estão sendo estudadas e já colocadas em prática são: contenção em rios e barragens, recifes artificiais, tapetes reforçados, móveis e utensílios domésticos, enriquecimento do asfalto, sandálias e solados de sapatos, aterros reforçados, desvulcanização, barreiras e barricadas em estradas, coprocessamento na indústria cimenteira (combustível), co-processamento com xisto (Petrobrás SIX), combustível em usinas termelétricas e diversos tipos de pirólise. ${ }^{4,6,11}$

Diante disso, muitos estudos sobre a reutilização de pneus vêm sendo desenvolvidos por pesquisadores do mundo inteiro. $^{12-15}$ Entretanto, até 0 presente momento, não foram identificados relatos sobre o uso de rejeitos de pneus para a produção de resinas adsorventes. No presente trabalho, propomos a síntese de um novo material adsorvente obtido a partir de resíduos de pneus. Assim, objetiva-se a transformação de resíduos da indústria em materiais de importância tecnológica com valor agregado. Os resultados obtidos se destacam como uma alternativa para 0 tratamento de efluentes com características diversas. 


\section{Procedimento Experimental}

\subsection{Síntese da Resina}

O preparo da resina consistiu na sulfonação de $25 \%$ dos rejeitos de pneu em pó. O preparo do agente sulfonante foi realizado com a mistura de $9 \mathrm{~mL}$ de ácido sulfúrico e $17 \mathrm{~mL}$ anidrido acético num béquer mantido em um banho de gelo e sob agitação. Neste procedimento, $5 \mathrm{~g}$ do resíduo de pneu foi solubilizado parcialmente em 200 $\mathrm{mL}$ de hexano e, posteriormente, o agente sulfonante foi adicionado lentamente à solução, sob agitação constante. A mistura foi mantida em um sistema de refluxo a $50{ }^{\circ} \mathrm{C}$ durante 4 horas, sendo a reação interrompida com a adição de metanol. A resina foi recolhida mediante a centrifugação da solução resultante e em seguida a resina foi levada para uma estufa a $50{ }^{\circ} \mathrm{C}$ durante 6 horas. Esta metodologia e o produto foram patenteados pelos autores. ${ }^{16}$

\subsection{Caracterização do material}

A resina obtida foi analisada por microscopia eletrônica de varredura (MEV). Para obtenção das micrografias, as amostras foram montadas em suporte stubs, com fita de carbono dupla face e colocadas sobre uma película de papel alumínio. Em seguida, as amostras foram cobertas com ouro em evaporador Balzers SCD 050 e observadas em microscópio eletrônico de varredura Leo Evo
40XVP. Os cálculos de porosidade foram realizados pelo tratamento das imagens obtidas por MEV com aumento de $5000 \mathrm{x}$, utilizando o software Quantikov. ${ }^{17}$ As análises de espectroscopia na região do infravermelho com transformada de Fourier com refletância total atenuada (FTIR-ATR) foram realizadas com um espectrofotômetro Digilab Excalibur - FT3000. Análises termogravimétricas (SDT simultaneous TGADTA modelo TG Shimadzu MOD 8065 D1) foram efetuadas em atmosfera de ar com taxa de aquecimento de $10{ }^{\circ} \mathrm{C} \mathrm{min}^{-1}$. As análises de calorimetria exploratória diferencial (DSC) foram realizadas em um aparelho da marca Rigaku MOD8065 D1, utilizando termistor de $\mathrm{Pt}$ e $\mathrm{Al}_{2} \mathrm{O}_{3}$ como material de referência. Utilizou-se cerca de 5 $\mathrm{mg}$ das amostras, que foram aquecidas continuamente com temperatura variando de 30 a $600{ }^{\circ} \mathrm{C}$, em atmosfera de ar com uma taxa de aquecimento de $10^{\circ} \mathrm{C} \mathrm{min}^{-1}$.

\subsection{Teste de adsorção de corantes}

O azul de metileno (AM) e o vermelho reativo (VR) foram utilizados como compostos modelos nos testes de adsorção devido ao fato de que estes compostos apresentam uma forte absorção na região visível $\left(\lambda_{\max }=645,0\right.$ e $540,0 \mathrm{~nm}$, respectivamente) e uma elevada solubilidade em água e propriedades semelhantes àquelas apresentadas pelos corantes têxteis, que são de difícil degradação. As estruturas dos corantes AM e VR são apresentadas na Figura 1.

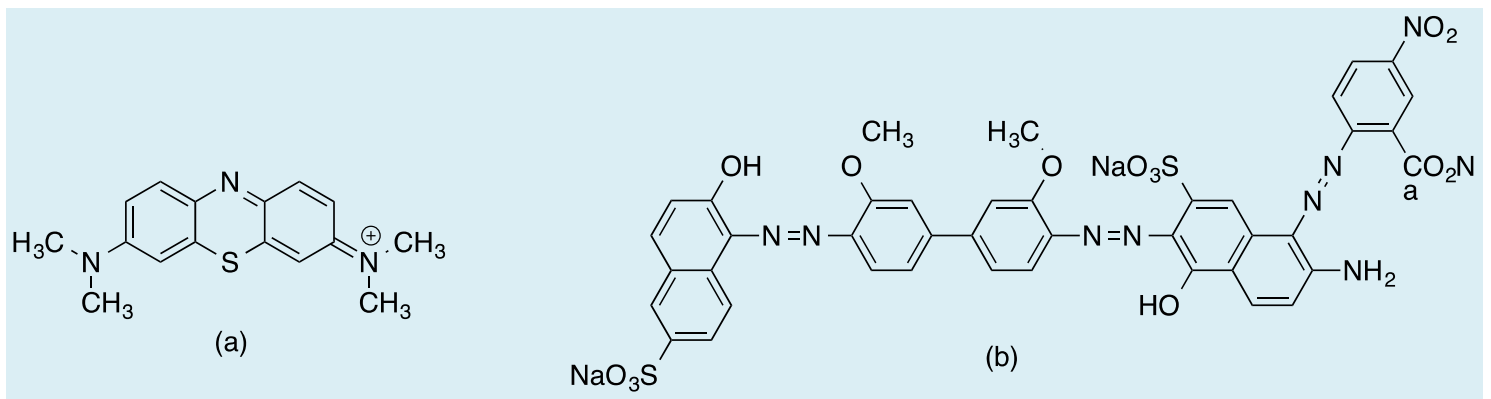

Figura 1. Estruturas dos corantes orgânicos: (a) azul de metileno e (b) vermelho reativo 
Para a obtenção das isotermas de adsorção, os testes foram feitos em bateladas, a partir de $10 \mathrm{~mL}$ de soluções de AM e VR nas concentrações 10, 50, 100, 250 e $500 \mathrm{ppm}$ e em contato com $10 \mathrm{mg}$ da resina sob agitação constante, por um período de 24 horas. Os experimentos foram realizados em pH 10 com temperatura constante de 25 ${ }^{\circ} \mathrm{C}$. Após o tempo de contato, as soluções foram centrifugadas e o sobrenadante foi analisado por espectrofotometria de UVVisível (Shimadzu-UV-1601 PC), monitorandose a adsorção dos corantes AM e VR nos comprimentos de onda 645 e $540 \mathrm{~nm}$, respectivamente.

\subsection{Testes de adsorção de metais}

Os testes de adsorção foram realizados em batelada a temperatura ambiente. Foram adicionados $50 \mathrm{mg}$ da resina em $100 \mathrm{~mL}$ de solução contendo íons metálicos, preparados com concentrações de 0,1 e 0,01 mol L L dissolução dos respectivos sais $\mathrm{ZnCl}_{2}$ (Synth), $\mathrm{CuCl}_{2}$ (Ecidra), $\mathrm{FeCl}_{3}$ (Ecidra) e $\mathrm{K}_{2} \mathrm{CrO}_{4}$ (Reagen) em água destilada. Após $24 \mathrm{~h}$, tempo suficiente para atingir o equilíbrio de adsorção, as misturas foram centrifugadas. Em seguida, cada amostra foi centrifugada e uma alíquota de $3 \mathrm{~mL}$ foi retirada. Finalmente, as soluções foram analisadas utilizando um espectrofotômetro de absorção atômica (EAA), Varian, para determinar a concentração de metal residual. No intuito de determinar os limites do novo material desenvolvido, é importante ressaltar que as concentrações empregadas foram elevadas. Esta metodologia é bem estabelecida na literatura. ${ }^{18}$

\section{Resultados e Discussão}

Nessa parte do trabalho serão apresentados e discutidos os resultados obtidos em relação ao preparo e a aplicação da resina na adsorção de contaminantes em meio aquoso. Os testes avaliaram o potencial de adsorção da resina em meio aquoso na remoção de corantes têxteis (catiônicos e aniônicos) e metais pesados, tais como cromo, cobre, ferro e zinco.

\subsection{Caracterização do material sintetizado}

A resina foi caracterizada por diferentes técnicas destinadas a uma melhor compreensão das propriedades físicoquímicas deste novo material. A caracterização morfológica e estrutural foi realizada por MEV, como mostrada na Figura 2. Em particular, o desenvolvimento de novos materiais exigem um número de informações sobre as características microestruturais, no intuito, de entender as correlações entre a microestrutura, defeitos e propriedades. ${ }^{19}$ Uma dificuldade óbvia na caracterização realizada por MEV é que as imagens geradas são bidimensionais, enquanto que as estruturas que elas representam são tridimensionais. As micrografias apresentadas na Figura 2 mostram que as microestruturas são constituídas de partículas agregadas com superfícies similares. Além disso, a porosidade total apresentada foi de $14 \%$ para o resíduo de pneu em pó e de $17 \%$ para resina obtida após a modificação química. Estes resultados evidenciam um aumento da porosidade após a modificação química realizada, conferindo um aumento na área superficial da resina. Entretanto, uma descrição quantitativa da distribuição de poros através do tratamento digital das imagens em duas ou três dimensões é bastante complexa. Apesar de a porosidade ser uma característica muito importante no estudo de adsorção, é importante ressaltar que os resultados de MEV fornecem uma informação semiquantitativa sobre a formação de poros, a partir do tratamento digital das imagens obtidas. Outro aspecto relevante a ser citado, é o elevado rendimento da síntese que chega a ser superior a $85 \%$. 


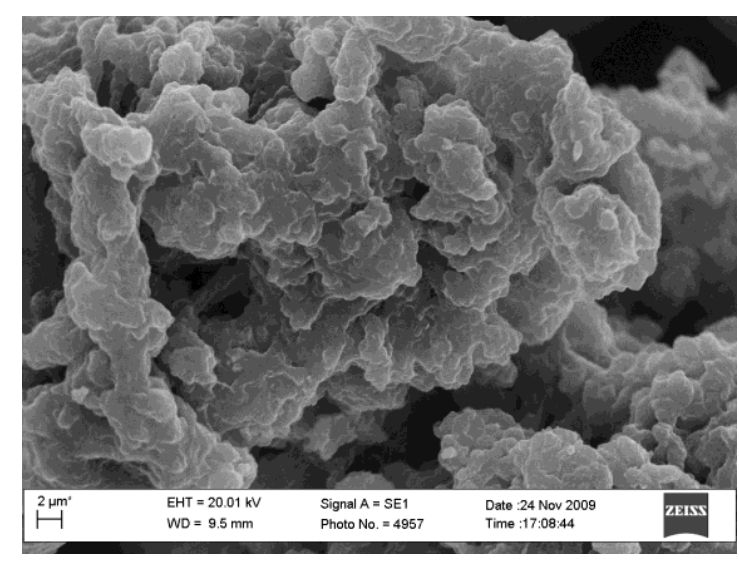

(a)

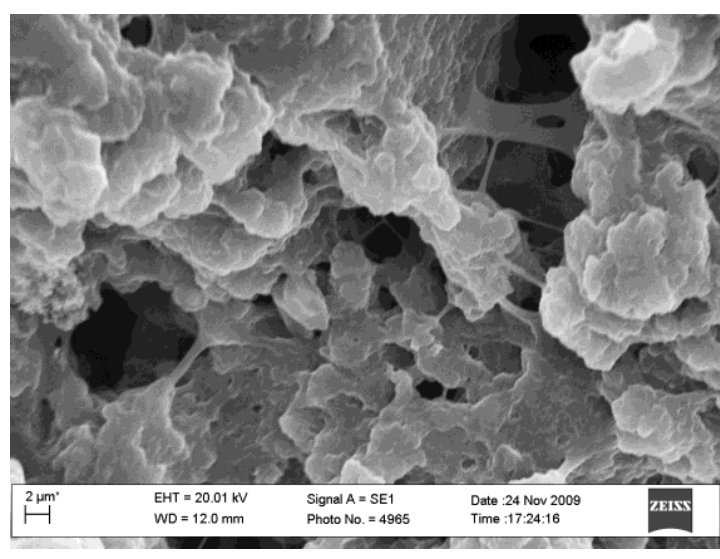

(b)

Figura 2. Imagens de MEV dos materiais: (a) do resíduo de pneu em pó e (b) da resina obtida após modificação química

A fim de analisar os grupos superficiais presentes na resina e avaliar a eficácia do processo de sulfonação realizado, utilizamos a técnica FTIR. Pela espectroscopia na região do infravermelho, observaram-se picos em torno de $4000-400 \mathrm{~cm}^{-1}$ (Figura 3). Mais especificamente, picos em torno de 3364 e 1628 sugerem a presença de grupos $\mathrm{OH}$, e picos em torno de 1148 e $1031 \mathrm{~cm}^{-1}$ relacionados ao estiramento so indicam características de grupos sulfônicos que foram gerados no material. ${ }^{20}$ As bandas fracas de absorção em $3500 \mathrm{~cm}^{-1}$ podem ser atribuídas à vibração de alongamento e de flexão dos grupamentos $\mathrm{OH}$ da água adsorvida na superfície da resina. A banda em $2331 \mathrm{~cm}^{-1}$ pode ser atribuída ao $\mathrm{CO}_{2}$ presente do ar. Esses resultados estão de bom acordo com a literatura. ${ }^{21}$

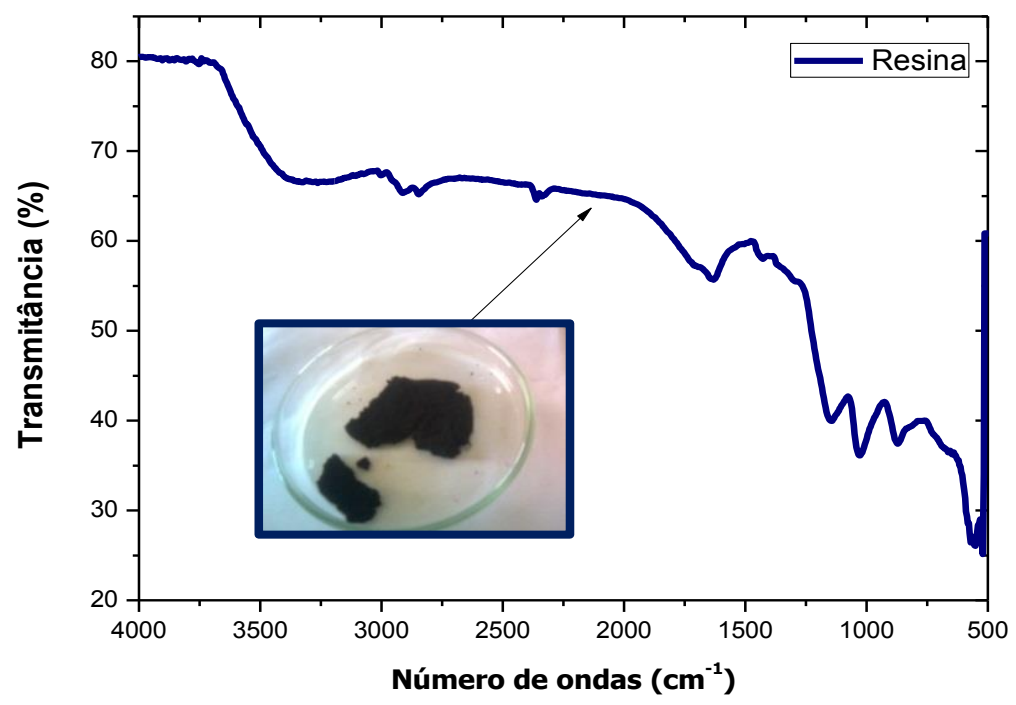

Figura 3. Espectro na região do infravermelho da resina

A análise termogravimétrica (TG em ar) e a calorimetria exploratória diferencial (DSC em ar) foram utilizadas para monitorar o perfil de estabilidade térmica após a 
modificação química da resina (Figuras 4 e 5). Os resultados obtidos da análise termogravimétrica do resíduo de pneu em pó (a) e da resina obtida após a modificação química (b) estão apresentados na Figura 4.

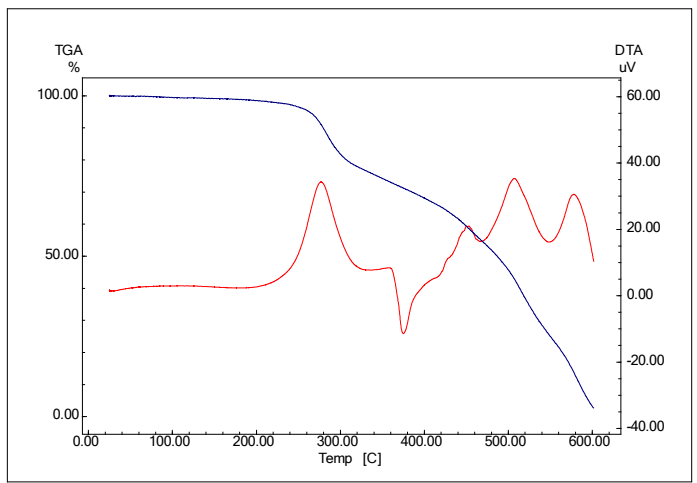

(a)

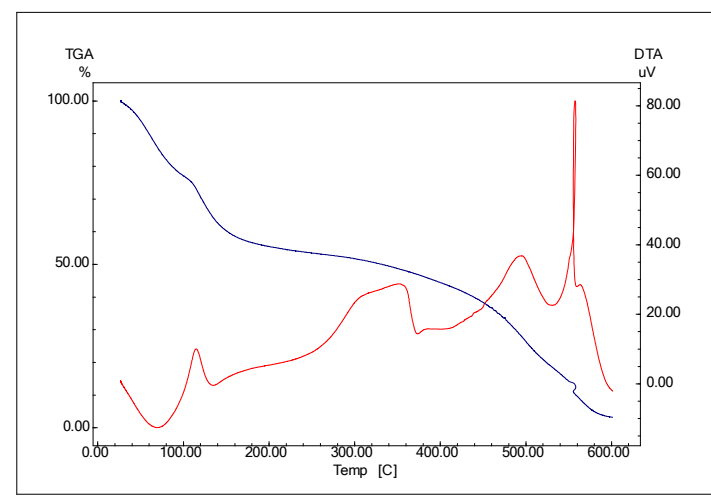

(b)

Figura 4. Análise termogravimétrica (TG) e em ar: (a) do resíduo de pneu em pó e (b) da resina obtida após modificação química

A partir das análises por TG, observa-se que a resina sofreu uma perda brusca de massa em cerca de $50 \%$ até $100{ }^{\circ} \mathrm{C}$ (Figura 4 b). Apesar dessa diferença relativa, no início da perda de massa com temperatura de decomposição entre $300-600{ }^{\circ} \mathrm{C}$, em ambos gráficos, pode-se observar picos comuns exotérmicos. É interessante observar também que ocorreu perda de $100 \%$ de massa a partir de $600{ }^{\circ} \mathrm{C}$, mostrando claramente que não houve a formação de cinzas, e evidenciando que o material obtido possui elevado teor de carbono, o que é desejável para aplicação como adsorventes. As análises de calorimetria exploratória diferencial (DSC em ar) estão apresentadas na Figura 5.
As medidas de DSC foram realizadas para analisar os eventos relacionados às mudanças físicas ou químicas baseadas na diferença de entalpia entre a amostra e o material de referência. Como observado nos resultados de DSC, a energia envolvida no processo endotérmico de perda de massa na região da temperatura entre $20^{\circ} \mathrm{C}$ a $120^{\circ} \mathrm{C}$ ocorreu devido às alterações químicas realizadas. Os valores obtidos durante a degradação térmica do pneu e da resina consistem de uma mistura muito complexa de compostos orgânicos de 5-20 átomos de carbono com uma proporção elevada de compostos aromáticos. A fração de gás é composta principalmente de $\mathrm{CO}, \mathrm{CO}_{2}, \mathrm{H}_{2}$ e hidrocarbonetos leves. ${ }^{22}$ 


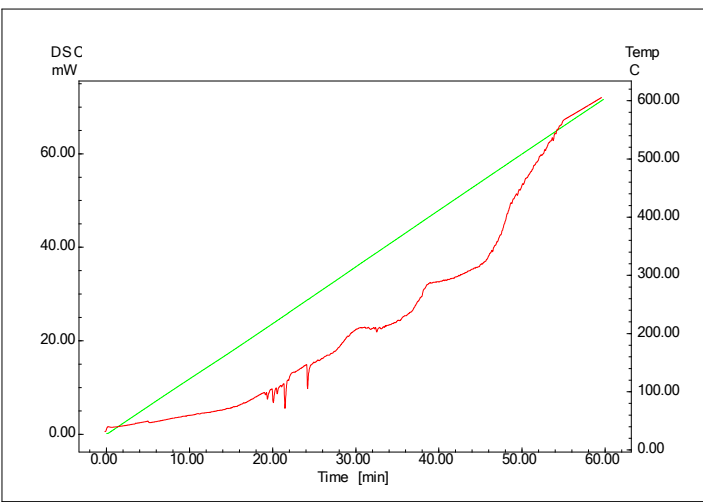

(a)

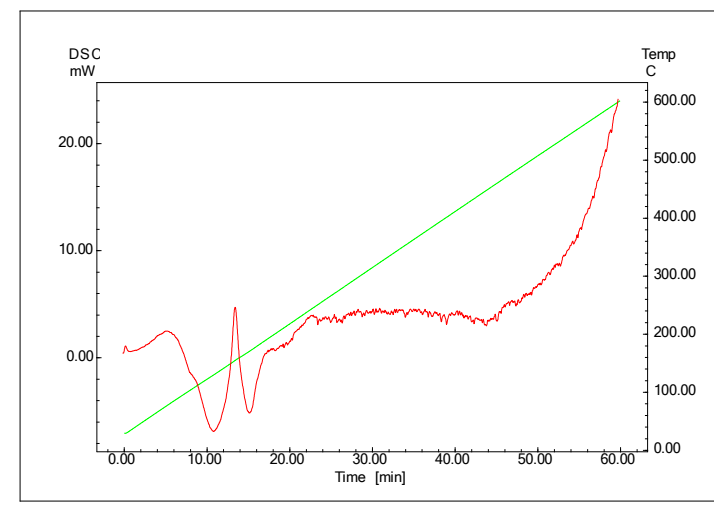

(b)

Figura 5. Análises de calorimetria exploratória diferencial (DSC) em ar: (a) do resíduo de pneu em pó e (b) da resina obtida após modificação química

A capacidade de adsorção da resina foi testada utilizando-se estes dois corantes orgânicos, sendo um catiônico (AM) e o outro aniônico (VR). A quantidade de corante adsorvida pelo sólido, $\mathrm{q}_{\mathrm{e}}\left(\mathrm{mg} \mathrm{g}^{-1}\right)$, foi calculada empregando-se a Equação 1:

Nesta equação, Ci e Ce são as concentrações iniciais e de equilíbrio do corante na fase líquida, respectivamente $(\mathrm{mg}$ $\left.\mathrm{L}^{-1}\right), \mathrm{V}_{0}$ volume de solução $(\mathrm{L})$ e $\mathrm{W}$ a massa de sólido $(\mathrm{g}) .^{23}$ As isotermas de adsorção são apresentadas na Figura 6.

$$
\mathrm{q}_{\mathrm{e}}=\frac{V(\mathrm{Ci}-\mathrm{Ce})}{W} \quad \text { Equação } 1
$$

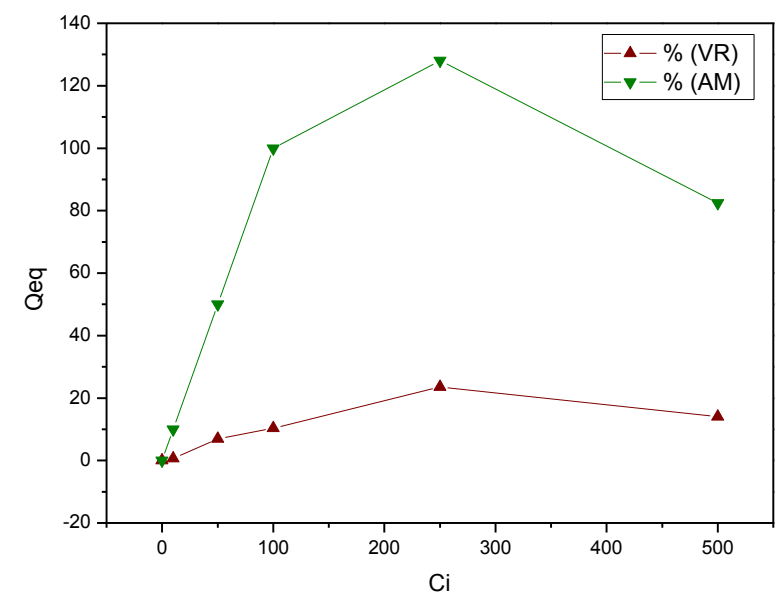

Figura 6. Isoterma de adsorção do AM (Azul de metileno) e VR (vermelho reativo)

A isoterma (Figura 6) demonstra a capacidade de adsorção da resina para os corantes em questão na temperatura de $25^{\circ} \mathrm{C}$. Os melhores resultados de adsorção foram para as soluções contendo $250 \mathrm{ppm}$. A capacidade máxima de adsorção de AM e VR foram avaliadas para a resina, obtendo os valores de 505,05 e 104,38 $\mathrm{mg} \mathrm{g}^{-1}$, respectivamente. 
O fato de a resina adsorver cinco vezes mais AM em relação ao VR pode ser atribuído às cargas dos grupos adicionados (grupo sulfônico) durante a etapa de modificação química do material. Estes resultados caracterizam este novo material como uma resina catiônica, apresentando potenciais aplicações no tratamento de efluentes com características diversas. Desta maneira, o material obtido também foi empregado na remoção de metais em meio aquoso. Para a realização dos testes, empregaram-se soluções contendo os íons $\mathrm{Cr}^{+3}, \mathrm{Cu}^{+2}, \mathrm{Zn}^{+2}$ e $\mathrm{Fe}^{+3}$ com concentrações variadas de 0,01 a $0,1 \mathrm{~mol} \mathrm{~L}^{-1}$. As análises foram realizadas em um espectrofotômetro de absorção atômica de chama, o que permitiu a quantificação dos metais adsorvidos pela resina, conforme apresentado na Tabela 1.

Tabela 1. Avaliação da remoção de metais em solução aquosa

\begin{tabular}{cc}
\hline Metal & $\begin{array}{c}\text { Capacidade de remoção de metal } \\
\text { (mg metal/g de adsorvente) }\end{array}$ \\
\hline $\mathrm{Cr}^{+3}$ & 55,25 \\
$\mathrm{Zn}^{+2}$ & 3,96 \\
$\mathrm{Cu}^{+2}$ & 43,29 \\
$\mathrm{Fe}^{+3}$ & 161,29 \\
\hline
\end{tabular}

Pode-se observar que a resina apresenta elevada capacidade de remoção de cátions em solução aquosa. Esta elevada capacidade de remoção de cátions se deve ao grupo sulfônico adicionado, que possibilita uma considerável adsorção em virtude da atração eletrostática desse grupo com os cátions em solução. Os resultados apresentados na Tabela 1 mostram que a resina apresentou uma maior capacidade de remoção de cátions trivalentes em relação aos bivalentes, sendo a seguinte ordem de estabilidade obtida para os nossos estudos: $\mathrm{Fe}^{3+}>\mathrm{Cr}^{3+}>$ $\mathrm{Cu}^{2+}>\mathrm{Zn}^{2+}$. A capacidade de adsorção do $\mathrm{Fe}^{+3}$ é cerca de três vezes maior do que a do $\mathrm{Cr}^{+3}$, o que sugere que este metal forma um complexo mais estável com o material adsorvente.

É importante ressaltar que neste trabalho nenhum estudo foi realizado para avaliar o efeito do $\mathrm{pH}$ e da temperatura no processo de adsorção destes materiais, uma vez que estas variáveis afetam diretamente as propriedades de adsorção. ${ }^{24-26} \mathrm{Em}$ particular, quanto mais ácido for o meio, menor a chance dos grupos sulfônicos da resina estarem desprotonados, tendo em vista os baixo valores de pKa encontrados para estes grupos, provocando desta maneira uma diminuição das forças atrativas ao longo do processo de adsorção e reduzindo a capacidade de remoção destes cátions pelo novo material.

Estudos teóricos prévios ${ }^{27,28}$ relatam a estabilidade, o mecanismo de formação e a reatividade de uma série de complexos metálicos envolvendo grupos fosfatos, carboxílicos e nitrogenados como ligantes. Nestes estudos, foram empregadas várias metodologias teóricas baseadas no método DFT (sigla que vem do inglês e significa Teoria do Funcional de Densidade) que apresentaram uma boa concordância com os resultados experimentais encontrados na literatura. Desta forma, a mudança de tendência está provavelmente relacionada com a diferença do grupo presente na superfície, sugerindo que diferentes modificações químicas permitem o planejamento de materiais adsorventes que possam ser mais seletivos a um determinado tipo em específico de metal. Novos estudos estão em progresso no intuito de confirmar estas hipóteses.

\section{Conclusão}

A síntese de um novo material adsorvente 
obtido a partir de resíduos de pneus foi proposta. As medidas de MEV, FTIR, TG, DSC, UV-vis e espectrofotômetro de absorção atômica de chama foram utilizadas extensivamente na análise e racionalização das observações experimentais, bem como na investigação estrutural desta nova resina adsorvente com grande potencial a ser utilizado na remoção de contaminantes em água com características diversas. A facilidade do processo de obtenção da resina, a eficiência da resina quanto à capacidade de adsorção e o seu baixo custo indicam que o material apresentado neste trabalho pode ter grande importância ambiental e tecnológica. O grupo sulfônico adicionado ao resíduo de pneu possibilitou uma considerável adsorção em virtude da atração eletrostática com as moléculas de corantes e os cátions em solução. Outro aspecto marcante deste estudo consiste no fato de que a resina pode ser facilmente removida do meio aquoso por separação mecânica após a adsorção, assim o adsorvente pode ser reutilizado, caracterizando um processo mais eficaz e menos oneroso.

Este trabalho buscou avaliar o emprego de uma nova resina, a base de rejeitos de pneu, na remoção de contaminantes em solução aquosa. Este novo material tem potencial para contribuir com a redução de impactos ambientais gerados pelo descarte indiscriminado de efluentes nos meios aquosos e resíduos sólidos, minimizando, assim, os custos com os processos de tratamento dos rejeitos a serem descartados ou reutilizados nos processos de produção. Desta maneira, a transformação de resíduos ou rejeitos em materiais de importância tecnológica e com valor agregado desponta como uma alternativa bastante promissora para os mais diversos tipos de resíduos.

\section{Agradecimentos}

Os autores agradecem a CAPES, CNPq e a FAPEMIG pelo suporte financeiro e ao Central de Análise e Prospecção Química da Universidade Federal de Lavras (CAPQ/UFLA), onde foi possível a realização das análises.

\section{Referências Bibliográficas}

${ }^{1}$ Rodolfo Jr, A. ; Nunes L. R.; Ormanji, W.; Tecnologia do PVC, 2a. ed., Braskem: São Paulo, 2006.

${ }^{2}$ Hage Jr., E. Aspectos Históricos sobre o Desenvolvimento da Ciência e da Tecnologia de Polímeros. Polímeros 1998, 8, 6. [CrossRef]

${ }^{3}$ Cordebello, F. S. Polímeros do Futuro Tendências e Oportunidades: Palestras Técnicas (II). Polímeros 2003, 13, E4. [CrossRef]

${ }^{4}$ Martínez, J. D.; Murillo, R.; Puy, N.; García, T.; Navarro, M. V.; Mastral, A. M. Waste tyre pyrolysis - A review. Renewable and Sustainable Energy Reviews 2013, 23, 179. [CrossRef]

${ }^{5}$ Nohara, J. J.; Acevedo, C. R.; Pires, B. C. C.; Corsino, R. M. GS-40 - Resíduos Sólidos: passivo ambiental e reciclagem de pneus. Thesis 2005, 3, 21. [Link]

${ }^{6}$ Lagarinhos, C. A. F.; Tenório, J. A. S. Tecnologias Utilizadas para a Reutilização, Reciclagem e Valorização Energética de Pneus no Brasil. Polímeros 2008, 18, 106. [CrossRef]

${ }^{7}$ CONSELHO NACIONAL DO MEIO AMBIENTE - CONAMA. Resolução CONAMA no 416, de 30 de setembro de 2009. Dispõe sobre a prevenção à degradação ambiental causada por pneus inservíveis e sua destinação ambientalmente adequada, e dá outras providências. [Link]

${ }^{8}$ Cimino, M. A.; Zanta, V. M. Gerenciamento de Pneumáticos Inservíveis (GPI): Análise crítica de ações institucionais e tecnologias para minimização. Engenharia Sanitária $e$ Ambiental 2005, 10, 299. [CrossRef]

${ }^{9}$ Nóbrega, R. P.; Tese de doutorado, Universidade de São Paulo, Brasil, 2007. [Link]

${ }^{10}$ Lemieux, P. M.; Lutes, C. C.; Santoianni, D. A. Emissions of organic air toxics from open 
burning: a comprehensive review. Progress in Energy and Combustion Science 2004, 30, 1. [CrossRef]

${ }^{11}$ Bernardo, M.; Lapa, N.; Gonçalves, M.; Mendes, B.; Pinto, F.; Fonseca, I.; Lopes, H. Physico-chemical properties of chars obtained in the co-pyrolysis of waste mixtures. Journal of Hazardous Materials 2012, 219, 196. [CrossRef] [PubMed]

${ }^{12}$ Williams, P. T.; Besler, S.; Taylor, D. T. The pyrolysis of scrap automotive tyres: The influence of temperature and heating rate on product composition. Fuel 1990, 69, 1474. [CrossRef]

${ }^{13}$ Sinn, H.; Kaminsky, W.; Janning, J. Processing of Plastic Waste and Scrap Tires into Chemical Raw Materials, Especially by Pyrolysis. Angewandte Chemie International Edition in English 1976, 15, 660. [CrossRef]

${ }^{14}$ Farcasiu, M. Another use for old tires. ChemTech 1993, 23, 22. [Link]

${ }^{15}$ Mis-Fernández, R.; Rios-Soberanis, C. R.; Arenas-Alatorre, J.; Azamar-Barrios, J. A. Synthesis of carbon nanostructures from residual solids waste tires. Journal of Applied Polymer Science 2012, 123, 1960. [CrossRef]

${ }^{16}$ La Porta, F. A.; Ramalho, T. C.; Silva, J. O.; $B R$ PI1100046 A2 2011. [Link]

${ }^{17}$ Pinto, L. C. M.; Tese de Doutorado, Universidade de São Paulo, Brasil, 1996. [Link]

${ }^{18}$ Carvalho, H. W. P.; Batista, A. P. L.; Hammer, P.; Luz, G. H. P.; Ramalho, T. C. Removal of metal ions from aqueous solution by chelating polymeric hydrogel. Environmental Chemistry Letters 2010, 8, 343. [CrossRef]

${ }^{19}$ La Porta, F. A.; Ferrer, M. M.; de Santana, Y. V. B.; Raubach, C. W.; Longo, V. M.; Sambrano, J. R.; Longo, E.; Andres, J.; Li, M. S.; Varela, J. A. J. Synthesis of wurtzite ZnS nanoparticles using the microwave assisted solvothermal method. Journal of Alloys and Compounds 2013, 556, 153. [CrossRef]

${ }^{20}$ Silverstein, R. M.; Spectrometric Identification of Organic Compounds, 7a. ed., Wiley: New York, 1998.
${ }^{21}$ Hampton, R. R. Applied Infrared Spectroscopy in the Rubber Industry. Rubber Chemistry and Technology 1972, 45, 546. [CrossRef]

${ }^{22}$ Díez, C.; Martínez, O.; Calvo, L. F.; Cara, J.; Morán, A. Pyrolysis of tyres. Influence of the final temperature of the process on emissions and the calorific value of the products recovered. Waste Management 2004, 24, 463. [CrossRef] [PubMed]

${ }^{23}$ Giles, C. H.; MacEwan, T. H.; Nakhwa, S. N.; Smith, D. Studies in adsorption. Part XI. A system of classification of solution adsorption isotherms, and its use in diagnosis of adsorption mechanisms and in measurement of specific surface areas of solids. Journal of the Chemical Society 1960, 3973. [CrossRef]

${ }^{24}$ Gonçalves, M.; Oliveira, L. C. A.; Guerreiro, M. C. Nióbia magnética como adsorvente de contaminantes orgânicos em meio aquoso: efeito da temperatura e do pH. Química Nova 2008, 31, 518. [CrossRef]

${ }^{25}$ Lin, Y.; Chen, Q.; Luo, H. Preparation and characterization of $\mathrm{N}$-(2carboxybenzyl)chitosan as a potential $\mathrm{pH}$ sensitive hydrogel for drug deliver. Carbohydrate Research 2007, 342, 87. [CrossRef] [PubMed]

${ }^{26}$ Magriotis, Z. M.; Sales, P. F.; Ramalho, T. C.; Rocha, M. V. J.; Leal, P. V. B. Influence of $\mathrm{pH}$ and of the Interactions Involved in Etheramine Removal in Kaolinite: Insights about Adsorption Mechanism. The Journal of Physical Chemistry C 2013, 117, 21788. [CrossRef]

${ }^{27}$ Caetano, M. S.; Ramalho, T. C.; Botrel, D. F.; Cunha, E. F. F.; Mello, W. C. Understanding the inactivation process of organophosphorus herbicides: A DFT study of glyphosate metallic complexes with $\mathrm{Zn}^{2+}$, $\mathrm{Ca}^{2+}, \mathrm{Mg}^{2+}, \mathrm{Cu}^{2+}, \mathrm{Co}^{3+}, \mathrm{Fe}^{3+}, \mathrm{Cr}^{3+}$, and $\mathrm{Al}^{3+}$. International Journal of Quantum Chemistry 2012, 112, 2752. [CrossRef]

${ }^{28}$ La Porta, F. A.; Giacoppo, J. O. S.; Ramos, P. H.; Guerreiro, M. C.; Ramalho, T. C. Computational Insights into the Role of the Frontiers Orbital in the Chemistry of Tridentate Ligands. American Journal of Chemistry 2012, 2, 255. [CrossRef] 